This document is the accepted manuscript version of the following article:

Süess, M. J., Wolf, J. M., Jouy, P., Bonzon, C., Beck, M., Hundt, M., ... Faist, J. (2016).

Analysis of dual-section DFB-QCLs for spectroscopic applications. In 2016 compound

semiconductor week (CSW) (p. 7528846 (2 pp.). https://doi.org/10.1109/ICIPRM.2016.7528846

\title{
Analysis of dual-section DFB-QCLs for spectroscopic applications
}

\author{
Martin J. Süess ${ }^{1}$, Johanna M. Wolf ${ }^{1}$, Pierre Jouy ${ }^{1}$, Christopher Bonzon ${ }^{1}$, Mattias Beck ${ }^{1}$, Morten \\ Hundt $^{2}$, Béla Tuzson ${ }^{2}$, Lukas Emmenegger ${ }^{2}$, Jérôme Faist ${ }^{1}$ \\ 1 : Institute for Quantum Electronics, ETH Zürich, Ch-8093 Zürich, Switzerland \\ 2 : Laboratory for Air Pollution and Environmental Technology, EMPA, 8600 Dübendorf, Switzerland \\ martin.sueess@phys.ethz.ch
}

\begin{abstract}
We investigate the functionalities of dual-section (DS) distributed feedback (DFB) quantum cascade lasers (QCLs) in continuous wave (CW) and intermittent continuous wave (iCW) operation. We analyze internal etaloning with spectrally resolved light-current-voltage characterization, time-resolved spectral analysis of thermal chirping during iCW operation. The experimental analysis is accompanied by a combination of finite element method (FEM) simulations and transfer matrix method (TMM) calculations of DS-DFB structures. Finally, we demonstrate how the internal etaloning can be minimized by means of anti-reflective (AR) coatings and how spectroscopic data with stateof-the-art precision can be acquired with such lasers.
\end{abstract}

\section{INTRODUCTION}

QCLs are unipolar coherent light sources emitting in the mid-infrared (MIR) part of the electromagnetic spectrum. Their emission spectrum allows access to the fundamental absorption bands of many molecular species, allowing for gas concentration measurements with high precision and sensitivity by direct laser absorption spectroscopy. Using a single DFB-QCL we are usually limited to one or two species that can be targeted. Especially in environmental monitoring an ideal measurement station should be capable of simultaneously measuring multiple species in the same interaction volume. Yet, the technology transfer from single- to multi-gas requires the addition of other laser sources. However, adding independent laser sources to a system requires a beam combination scheme, independent driving electronics and temperature control. An interesting alternative to combining multiple laser beams are lasers able to emit more than one color in the same beam [1]. The thermal chirp during these pulses can then be used as a spectroscopic scan [2]. In this article we investigate the functionalities of DS-DFB QCLs operated in $\mathrm{CW}$ and $\mathrm{iCW}$.

\section{RESULTS}

DS-DFB lasers capable of lasing in CW operation at room temperature are used. A grating with quarter wave shift (QWS) enables single mode lasing close to $4.4 \mu \mathrm{m}$ and $4.6 \mu \mathrm{m}$. The LI curves in figure 1 show regularly spaced power fluctuations. We accredit these oscillations to internal etaloning caused by a temperature induced difference of the effective refractive index $n_{\text {eff }}$ between the active and the passive section. In order to better understand this effect, thermal 3D FEM simulations (figure 2) were used to calculate temperature profiles along the active region in the laser for different pumping currents $(\Delta \mathrm{I}=1 \mathrm{~mA})$. From this data the spatially and thermally dependent refractive index can be calculated and used in TMM calculations to predict the cavity losses of thermally influenced DS-DFB structures. The losses of the QWS mode and other interfering modes are approximately two orders of magnitude apart and their crossing points predicts a discrete hop between two modes (figure $3 \mathrm{a} \& \mathrm{~b}$ ). According to this simulation lasing on a mode with lower losses (e.g. the stopband edge) should be expected, which is in contrast to the experimental data (figure 3c), where only one single mode can be observed, which does not turn off or hop. We accredit this discrepancy to an over-simplification of the model and/or numerical uncertainties. Nonetheless, the spectral periodicity of the intensity modulation observed in experimental data (figure 3c) is well reproduced, confirming the hypothesis of an internal etalon caused by a temperature difference between the active and passive section.

In order to reduce the effect of the internal etalon the facets of such lasers were coated with AR coatings. Boxcar measurements were performed on the back laser of a DS-DFB QCL operated in $\mathrm{iCW}$, before and after the application of the AR coatings (figure 4a). The comparison of the intensity evolution during the pulse (figure $4 \mathrm{~b}$ ) shows that the effect of the internal etalon can be reduced significantly. Whereas the laser before coating temporarily turned off during the pulse (red curve), the amplitude fluctuations were reduced to maximally $15 \%$ after the application of the coating (green curve), without affecting the side mode suppression (figure 4a, inset). The residual intensity fluctuation is accredited to imperfections in the coating, which feature a reflectivity of 0.6 at the desired wavelength. Nonetheless, the AR coated DS-DFB QCLs are suitable for direct laser absorption spectroscopy of trace gases. We measured transmission spectra of air at $50 \mathrm{mbar}$ in a multipass cell recorded with both DFB sections of such a device, driven intermittently with $20 \mu$ s pulses at $10 \mathrm{kHz}$. We found the precision of the concentration measurements by measuring constant concentrations of the target species in air from a gas cylinder and determining the Allan-variance. Table 1 summarizes the found precisions for the gasses that we measured with a DS-DFB QCL. The here presented DS-DFB QCLs perform well enough to acquire concentration data with state-of-the-art precision.

1. Jagerska, J et al. Dual-wavelength quantum cascade laser for trace gas spectroscopy. Applied Physics Letters 2014, 105 .

2. Fischer, M. et al. Intermittent operation of qc-lasers for mid-ir spectroscopy with low heat dissipation: Tuning characteristics and driving electronics. Optics Express 2014, 22, 7014-7027. 


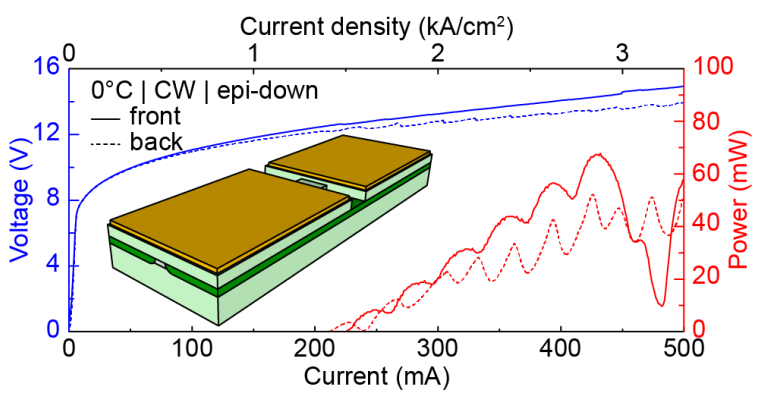

Fig.1: Light-current-voltage characteristic of a dual-section DFB QCL. Inset: Schematic drawing of a dual-section DFB QCL.

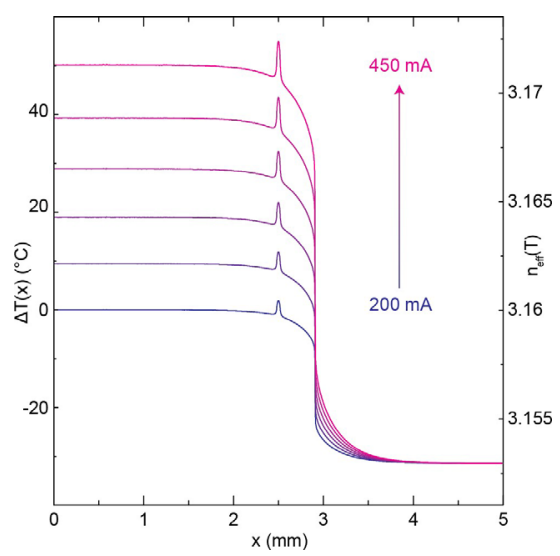

Fig. 2: FEM simulation of the temperature distribution inside the active region of a dual-section QCL at different operation currents. The right axis is the effective refractive waveguide index calculated from this distribution

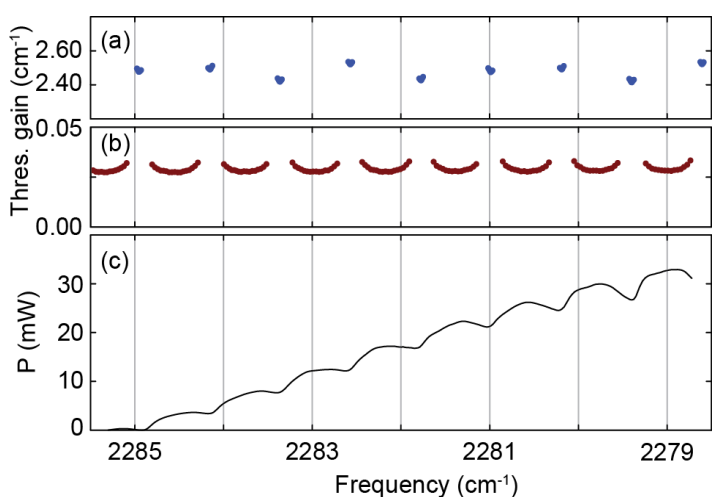

Fig. 3: Comparison between $(\mathrm{a} \& \mathrm{~b}) \mathrm{TMM}$ calculated modal losses and (c) a spectally resolved light-current characteristic.

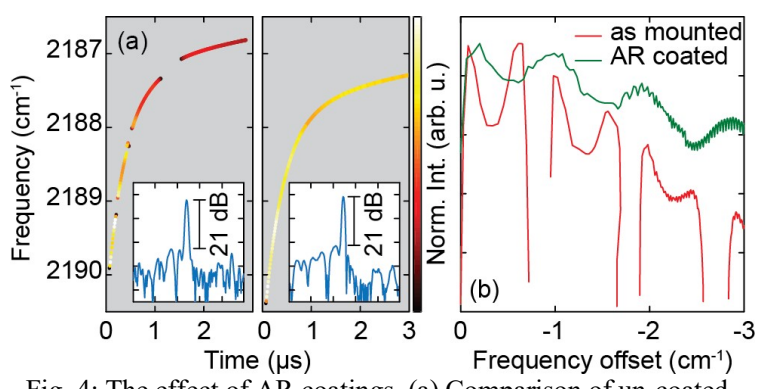

Fig. 4: The effect of AR coatings. (a) Comparison of un-coated (left) and coated (right) dual-section DFB in iCW operation. (b) Analysis of a the intensity recorded in the measurements displayed in (a).

Table 1: Precision of concentration measurements

\begin{tabular}{|c|c|c|}
\hline Gas & 1 s Noise & 60 s Noise \\
\hline $\mathrm{CO}$ & $0.8 \mathrm{ppb}$ & $0.17 \mathrm{ppb}$ \\
\hline $\mathrm{N}_{2} \mathrm{O}$ & $0.9 \mathrm{ppb}$ & $0.14 \mathrm{ppb}$ \\
\hline $\mathrm{CO}_{2}$ & $1.2 \mathrm{ppm}$ & $0.2 \mathrm{ppm}$ \\
\hline${ }^{13} \mathrm{CO}_{2} /{ }^{12} \mathrm{CO}_{2}$ & $3 \%$ & $0.5 \%$ \\
\hline
\end{tabular}

